\title{
Les maladies rares affectent 30 millions de personnes en Europe
}

Romain Lazor ${ }^{a}$,

Loredana D'Amato Sizonenko ${ }^{b}$

a membre du Comité scientifique Orphanet Suisse, coordinateur du Registre suisse des maladies rares pulmonaires (SIOLD)

b Coordinatrice Orphanet Suisse

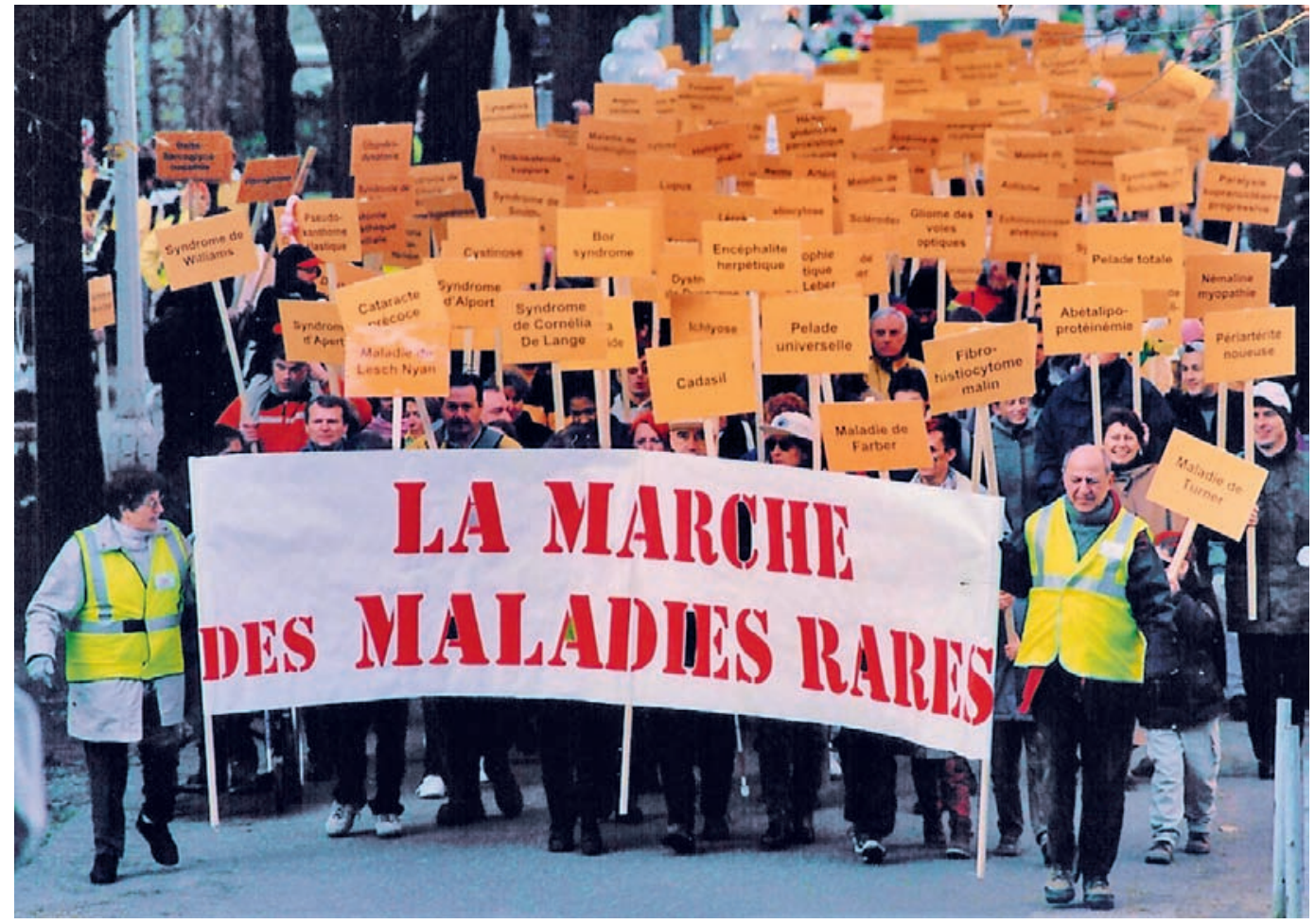

\section{Résumé}

En Europe, une maladie est dite rare lorsqu'elle affecte moins d'une personne sur 2000. C'est une affection invalidante pouvant mettre en jeu le pronostic vital et qui nécessite des efforts combinés spéciaux pour sa prise en charge. Plus de 7000 maladies rares ont été identifiées à ce jour. Prises dans leur ensemble, elles affectent 30 millions de personnes en Europe et constituent un problème majeur de santé publique. L'Union européenne et de nombreux Etats membres ont déjà pris des mesures spécifiques de grande ampleur pour lutter contre les maladies rares, mais cette problématique est encore largement méconnue en Suisse. La première Journée européenne des maladies rares invite les autorités politiques suisses, les professionnels de santé, les scientifiques et l'industrie pharmaceutique à soutenir les efforts déjà initiés et progresser dans la lutte contre les maladies rares dans notre pays.

\section{Introduction}

La première Journée européenne des maladies rares a eu lieu le 29 février 2008. Son but était d'informer et de sensibiliser le public et les institutions de santé à la problématique des maladies rares. Des conférences de presse, pétitions, marches, collectes, exposés, galas, concerts, interventions parlementaires et autres manifestations ont eu lieu dans de nombreux pays.

On dénombre aujourd'hui plus de 7000 maladies rares. $80 \%$ ont une origine génétique, les autres causes étant infectieuses, environnementales, auto-immunes, dégénératives ou tumorales. Malgré la faible prévalence de chacune d'entre elles, on estime qu'elles affectent collectivement 6 à $8 \%$ de la population, ce qui représente près de 30 millions de personnes en Europe. Prises dans leur ensemble, ces maladies constituent donc un enjeu majeur de santé publique, qui est encore largement méconnu en Suisse. 


\section{Qu'est-ce qu'une maladie rare?}

Une maladie rare est définie en Europe comme une affection invalidante ou menaçante pour la vie qui touche moins d'une personne sur 2000 et nécessite des efforts combinés spéciaux pour sa prise en charge. Ces maladies sont souvent chroniques, progressives, dégénératives et entraînent des handicaps qui altèrent la qualité de vie. Toutes les spécialités médicales sont concernées.

Quelle que soit leur maladie, les patients atteints d'une maladie rare doivent faire face aux mêmes difficultés: retards et erreurs de diagnostic, manque d'informations, souffrance psychologique liée à l'isolement et au manque d'espoir thérapeutique, absence de soutien pratique dans la vie quotidienne. Les maladies rares sont aussi insuffisamment connues des professionnels de santé. Il y a peu de données scientifiques, et les traitements ne sont pas bien définis voire inexistants. La recherche est peu soutenue et l'industrie pharmaceutique n'est pas motivée à développer des médicaments en raison du manque d'intérêt commercial que représente le petit nombre de patients concernés. Le terme «orphelin»a été utilisé pour exprimer le délaissement vécu par ces malades et le faible intérêt des institutions de santé et de l'industrie pharmaceutique.

\section{Quelles solutions?}

La première prise de conscience du problème des maladies rares est apparue aux Etats-Unis vers 1980, sous l'impulsion d'associations de patients qui voulaient obtenir de nouveaux médicaments pour ces maladies négligées par l'industrie pharmaceutique. Sous leur pression et celle de l'opinion publique, le gouvernement américain a adopté en 1983 une loi favorisant le développement de médicaments «orphelins» (Orphan Drug Act) en accordant diverses facilités à l'industrie: aides technique et financière, procédure d'acceptation accélérée par les autorités de surveillance, extension de la durée du brevet et réductions fiscales. Cette politique a porté ses fruits: en 25 ans, plus de 300 médicaments orphelins ont été développés et enregistrés aux Etats-Unis. Les associations de patients américaines se sont aussi regroupées en une puissante fédération (NORD), qui est devenue un groupe de pression très influent.

L'Europe a également pris conscience des difficultés rencontrées par les patients atteints de maladies rares, et en particulier de l'inégalité d'accès au diagnostic et au traitement en comparaison des maladies fréquentes. Depuis 1999, l'Union européenne a pris des mesures spécifiques pour lutter contre les maladies rares, qui ont été identifiées comme problème prioritaire de santé publique. Différents programmes ont été lancés, dont la réglementation européenne des médicaments orphelins, des programmes d'action communautaires et des financements spécifiques pour la recherche. Certains pays ont développé des plans nationaux pour les maladies rares. C'est notamment le cas de la France, qui a désigné par appel d’offres 132 centres de référence spécialisés dans la prise en charge de certaines maladies rares ou de groupes de maladies.

Une caractéristique importante et originale des progrès réalisés dans le domaine des maladies rares a été l'établissement d'un fort partenariat entre patients, associations, professionnels de santé et industrie pharmaceutique. Alors qu'un tel partenariat n'existe pratiquement pas pour les maladies fréquentes, il se révèle indispensable dans le domaine des maladies rares car les différents acteurs dépendent les uns des autres pour progresser.

\section{L'information: une nécessité}

L'un des problèmes majeurs des maladies rares est le manque d'information concernant les ressources disponibles. Initié en 1997 par la France et soutenu par la Commission européenne, Orphanet est devenu le portail européen de référence sur les maladies rares et les médicaments orphelins, avec plus de 25000 utilisateurs quotidiens. Sa $4^{\mathrm{e}}$ version vient d'être mise en ligne. Ce site internet en libre accès destiné à tous les publics et disponible en 6 langues fournit des informations et un répertoire des services spécifiques sur les maladies rares et les médicaments orphelins. Ses objectifs sont de contribuer à l'amélioration du diagnostic et de la prise en charge des patients atteints de maladies rares, d'accélérer la recherche, de renforcer les interactions entre partenaires et institutions concernés, et d'améliorer l'utilisation des ressources existantes. Orphanet évolue continuellement pour répondre aux besoins de ses utilisateurs. Il propose aujourd'hui une encyclopédie sur plus de 5000 maladies rares ainsi qu'un répertoire des consultations spécialisées, centres de référence, laboratoires de diagnostic, projets de recherche, essais cliniques, registres, réseaux de professionnels et associations de patients. Ce répertoire couvre 35 pays européens dont la Suisse. Des équipes nationales sont chargées de collecter les informations sur les services spécialisés dans leurs pays respectifs, en collaboration avec leur comité scientifique.

\section{Et la Suisse?}

Dans le domaine des maladies rares, la Suisse suit la trace de l’Union européenne avec un retard de 
quelques années. Il n'existe pratiquement aucune donnée épidémiologique sur les maladies rares, mais l'extrapolation à partir des chiffres européens suggère que 500000 personnes en Suisse pourraient être affectées. La complexité du système politique et sanitaire suisse, avec ses gouvernements cantonaux et ses zones linguistiques, ralentit les développements au niveau politique. Il n'existe pas encore de centres de référence. Il n'y a pas non plus de programmes de recherche ni de financements spécifiques, et seules des institutions privées comme le Téléthon soutiennent des recherches ciblées sur certaines maladies. Si le concept de médicament orphelin est reconnu par l'Institut suisse des produits thérapeutiques, il n'existe pas d'incitation financière au développement de tels médicaments. Il existe déjà une centaine d'associations suisses de patients, mais elles ne se sont pas encore constituées en alliance nationale. Orphanet Suisse (www.orpha-net.ch), lancé en 2001, vient de recevoir l'approbation de la Conférence suisse des directeurs cantonaux de la santé (CDS) et la plupart des cantons contribuent à son financement. Quelques réseaux de professionnels et des registres ont été développés, comme le groupe et le registre suisse des maladies rares pulmonaires (www.siold.ch), mais leurs activités doivent être mieux reconnues et soutenues.

Dans un souci d'équité, notre système de santé doit fournir à chaque patient des prestations de haute qualité, quelle que soit la fréquence de la maladie dont il est atteint. La première Journée européenne des maladies rares invite les institutions suisses à soutenir les efforts initiés au niveau national et à progresser dans la direction prise par de nombreux autres pays pour lutter contre les maladies rares.

\section{Liens utiles}

- Orphanet Europe (base de données sur les maladies rares et les médicaments orphelins): www.orpha.net

- Orphanet Suisse: www.orpha-net.ch

- OrphaNews Europe (bulletin électronique): www.orpha.net/actor//cgi-bin/OAhome.php?Ltr=EuropaNews

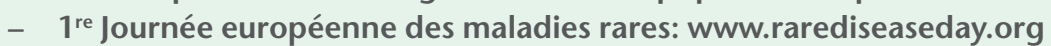

- Organisation européenne des maladies rares: www.eurordis.org

- Commission européenne, maladies rares: http://ec.europa.eu/health/ph_threats/non_com/rare_diseases_fr.htm

- Commission européenne, registre des médicaments orphelins: http://ec.europa.eu/enterprise/pharmaceuticals/register/index.htm

- France, Ministère de la santé, Plan national maladies rares: www.sante.gouv.fr/htm/dossiers/maladies_rares/plan.pdf

- National Institutes of Health, Office of Rare Diseases: http://rarediseases.info.nih.gov

- National Organization of Rare Disorders: www.rarediseases.org 